\title{
Airway Tree Extraction with Locally Optimal Paths
}

\author{
Pechin Lo ${ }^{1}$, Jon Sporring ${ }^{1}$, Jesper Johannes Holst Pedersen ${ }^{2}$, \\ and Marleen de Bruijne ${ }^{1,3}$ \\ 1 Image Group, Department of Computer Science, \\ University of Copenhagen, Denmark \\ pechin@diku.dk \\ 2 Department of Cardio Thoracic Surgery, Rigshospitalet - Copenhagen University \\ Hospital, Denmark \\ 3 Biomedical Imaging Group Rotterdam, Departments of Radiology \& Medical \\ Informatics, Erasmus MC, Rotterdam, The Netherlands
}

\begin{abstract}
This paper proposes a method to extract the airway tree from CT images by continually extending the tree with locally optimal paths. This is in contrast to commonly used region growing based approaches that only search the space of the immediate neighbors. The result is a much more robust method for tree extraction that can overcome local occlusions. The cost function for obtaining the optimal paths takes into account of an airway probability map as well as measures of airway shape and orientation derived from multi-scale Hessian eigen analysis on the airway probability. Significant improvements were achieved compared to a region growing based method, with up to $36 \%$ longer trees at a slight increase of false positive rate.
\end{abstract}

\section{Introduction}

Analysis of the airways in computed tomography (CT) is crucial for the understanding of various lung diseases [1. However due to the difficulties of the extraction process, the airways remain among the least understood structures in the lungs.

Most methods used for segmenting the airway tree from CT images are based on the concept of region growing [2 3 4 5]. The main problem with the standard region growing approach is that the segmentation may "leak" to surrounding lung parenchyma, if the contrast with the airway wall is low due to e.g. noise or pathology. Various strategies have been used to solve this problem, e.g. using geometric criteria to detect and remove leakage 234], or using improved appearance models to avoid leakage [5].

In this paper, we propose a new method for airway tree extraction method that continually extends the tree with locally optimal paths. The advantage of using such a path search approach is that the algorithm is able to look further ahead and can therefore overcome local occlusions. Occlusions may be caused for instance by noise or pathologies such as mucus plugging. Using the trained 
appearance model described in [5] as the basis for our cost function, the Dijkstra algorithm is applied within a sphere centered at a seed point to obtain candidate airway paths. Candidate paths that satisfy various criteria based on airway probability, shape, and orientation are retained, and potential bifurcation points and new end points are stored and subsequently used as new seed points. This process is repeated until no more valid candidate paths can be found.

The work is inspired by minimal path based approaches in vessel segmentation and diffusion tensor imaging (DTI) tractography (see e.g. 667]). These approaches are however typically either limited to extracting a single optimal path or require a user to specify the end points in advance. Another example is [8], where a complete vessel tree is extracted as a collection of optimal paths from a converged fast marching algorithm. In contrast to [8], we may be able to extract also less salient branches and thus find a more complete tree by searching for optimal paths locally and recursively. The notion of performing optimal path extraction locally was recently also proposed in [9], where various examples are shown in which a local approach outperforms the global approach. However, the focus of that paper was on extracting single paths or contours and the method is not capable of handling bifurcations.

Our work is similar in spirit to [10, which aims at reaching a more global solution for airway segmentation by detecting potential airway branches throughout the lungs with a tube detection scheme, and subsequently connecting these using a graph search method. However, our method is more flexible and can be customized to other tree segmentation tasks by simply modifying the cost function and the confidence measure.

\section{Tracking Locally Optimal Paths}

This section explains how the Dijkstra algorithm is applied locally and how the optimal paths are selected. We assume that a cost function $F$, a confidence measure $D$, and a list of initial candidate points are provided. A candidate point is a point that belongs to a previously extracted airway branch, and for which the departing paths are not yet extracted. Each candidate point is associated with the orientation and the average radius of the airway branch it belongs to.

At every iteration, a candidate point is taken from the list and evaluated. New paths extending from the candidate points are generated through a process of candidate path extraction and selection. Additional new candidate points from these new paths will then be added into the list. The iterative process ends once no more candidate points are available for evaluation.

\subsection{Extracting Candidate Paths}

Given a candidate point $\boldsymbol{x}_{0}$, with the branch orientation $\boldsymbol{d}_{0}$, optimal paths are computed from $\boldsymbol{x}_{0}$ to every point within a sphere of radius $r_{s}$ using the Dijkstra algorithm. We refer to these optimal paths as the candidate path from $\boldsymbol{x}_{0}$ to $\boldsymbol{x}$. The traveling cost between two neighboring voxels is computed using cost function $F$. 
Candidate paths are extracted for all points on the surface of the sphere that satisfy the following conditions:

1) Local minima: End points should form a local minimum, in terms of minimal cost from $\boldsymbol{x}_{0}$, on the surface of the sphere.

2) Departing angle: To limit the search to branches that do not deviate too much from the initial direction, end points should satisfy $L\left(\boldsymbol{x}-\boldsymbol{x}_{0}\right), \boldsymbol{d}_{0} \leq \alpha$, as shown in Fig. 1(c)

\subsection{Evaluating the Candidate Paths}

The most likely airway branches are selected from the extracted candidate paths by the following three selection criteria in a low to high cost order:

1) Confidence: The majority of points on a path must have high confidence measure $D$. Hence, we require the $N$ th percentile of the confidence measure of a path to be greater than $\beta$ in order to be selected.

2) Straightness: As airway branches are relatively straight in general, we require a path $C$ to satisfy $l_{\text {path }}(C)<\gamma l\left(\boldsymbol{x}_{0}, \boldsymbol{x}\right)$, where $l_{\text {path }}(C)$ is the length of the path $C$ and $l\left(\boldsymbol{x}_{0}, \boldsymbol{x}\right)$ is the distance from $\boldsymbol{x}_{0}$ to $\boldsymbol{x}$.

3) Non-overlap: Selected paths are not allowed to overlap each other and should be at least $\delta \mathrm{mm}$ apart. The distance between a candidate path and the previously selected paths is measured as the minimum distance between the end point of the candidate path and the points in the selected paths. We also ensure that a selected candidate path will not intersect with other paths obtained from previously evaluated candidate points. Prior to applying this criterion, an additional trimming process is added to remove low confidence points at the end of the path, which exist because of the used of fixed end points. The trimmed path is stored if the path is selected.

A maximum of $N_{\max }$ paths is retained.

\subsection{Updating the List of Candidate Points}

The end points of the newly selected paths are added to the list of candidate points. All potential bifurcation points, defined as points where candidate paths depart from a selected path, are also added to the list. The direction for each of the new candidate points is the direction of the selected path it belongs to.

\section{Cost Function}

Unlike most current airway segmentation methods [2/3/4/10] that use only image intensity, our proposed method operates on the soft classification resulting from a voxel classification based appearance model [5].

The appearance model uses a $K$ nearest neighbor (KNN) classifier that is trained to differentiate between voxels from airway and non-airway classes using various local image descriptors at different scales. To circumvent the requirement 
for high-quality ground truth segmentations of the complete airway tree, we follow the interactive training procedure described in [5]. A moderated KNN [1] output is used, where the posterior probability of a feature vector $\boldsymbol{f}(\boldsymbol{x})$, obtained at voxel position $\boldsymbol{x}$, to belong to the airway class is defined as

$$
P_{A}(\boldsymbol{f}(\boldsymbol{x}))=\frac{K_{A}(\boldsymbol{f}(\boldsymbol{x}))+1}{K+2},
$$

where $K_{A}(\boldsymbol{f}(\boldsymbol{x}))$ is the number of nearest neighbors around $\boldsymbol{f}(\boldsymbol{x})$ that belong to the airway class obtained from a total of $K$ nearest neighbors.

The airway probability $P_{A}(\boldsymbol{f}(\boldsymbol{x}))$ is used directly as the confidence measure $D$ in Section 2 to discard unlikely paths. The cost function $F$ is designed such that local paths are searched in the direction of probable airways, which appear as bright, cylindrical structures in $P_{A}$. The local orientation of the airways is derived through multi-scale Hessian eigen analysis on the airway probability map. The scale is selected for each voxel independently using the scale normalized [12] Frobenius norm of the Hessian matrix $\omega\left(\sigma_{i}\right)=\sigma_{i}^{2} \sqrt{\lambda_{1}\left(\sigma_{i}\right)^{2}+\lambda_{2}\left(\sigma_{i}\right)^{2}+\lambda_{3}\left(\sigma_{i}\right)^{2}}$, where $\left|\lambda_{1}\right| \geq\left|\lambda_{2}\right| \geq\left|\lambda_{3}\right|$ are the eigenvalues of the Hessian matrix. The local scale, $\sigma_{l}$, is then obtained as the smallest scale that corresponds to a local maximum of $\omega\left(\sigma_{i}\right)$ across a list of scales $\left\{\sigma_{\min }, \ldots, \sigma_{\max }\right\}$, where $\sigma_{\max }$ is chosen slightly larger than the current airway radius. A measure $M_{\text {tube }}$, indicating how well the surrounding image structure fits the model of a solid bright tube, can then be defined as

$$
M_{\text {tube }}(\boldsymbol{x})= \begin{cases}0, & \lambda_{1}\left(\sigma_{l}\right) \geq 0 \text { or } \lambda_{2}\left(\sigma_{l}\right) \geq 0, \\ \frac{\left|\lambda_{2}\left(\sigma_{l}\right)\right|-\left|\lambda_{3}\left(\sigma_{l}\right)\right|}{\left|\lambda_{2}\left(\sigma_{l}\right)\right|}, & \text { otherwise, }\end{cases}
$$

The orientation of the tube at $\boldsymbol{x}$ is given by $\boldsymbol{v}_{3}(\boldsymbol{x})$, which is the eigenvector corresponding to $\lambda_{3}\left(\sigma_{l}\right)$.

The cost function $F$ combines the airway probability, tubeness measure, and airway direction estimates as:

$$
F\left(\boldsymbol{x}_{s}, \boldsymbol{x}_{t}\right)=\frac{\left\|\boldsymbol{x}_{s}-\boldsymbol{x}_{t}\right\|_{2}}{\left|<\frac{\boldsymbol{x}_{s}-\boldsymbol{x}_{t}}{\left\|\boldsymbol{x}_{s}-\boldsymbol{x}_{t}\right\|_{2}}, \boldsymbol{v}_{3}\left(\boldsymbol{x}_{t}\right)>\right| P_{A}\left(\boldsymbol{f}\left(\boldsymbol{x}_{t}\right)\left(1+M_{\text {tube }}\left(\boldsymbol{x}_{t}\right)\right)\right.},
$$

where $\boldsymbol{x}_{s}$ and $\boldsymbol{x}_{t}$ is the source and target location. The cost $F\left(\boldsymbol{x}_{s}, \boldsymbol{x}_{t}\right)$ is low, when both the local airway probability is high and the propagation direction is parallel with the estimated airway orientation. The term with $M_{t u b e}$ is used to lower the cost further, when the local structure at $\boldsymbol{x}_{t}$ resembles a solid bright tube.

\section{Experiment and Results}

Experiments were conducted on low-dose CT images from the Danish Lung Cancer Screening Trial (DLCST) [13], where participants were current or former smokers at an age between 50-70 years. All images had a slice thickness of $1 \mathrm{~mm}$ and in-plane voxel size ranging from 0.72 to $0.78 \mathrm{~mm}$. A total of 32 


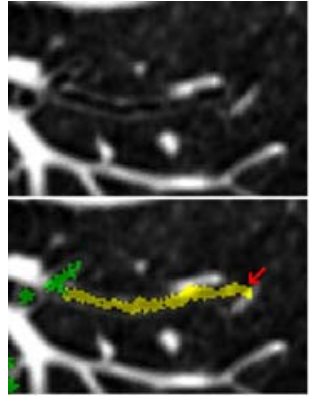

(a)

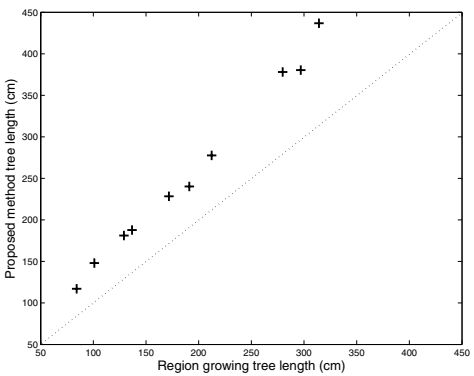

(b)

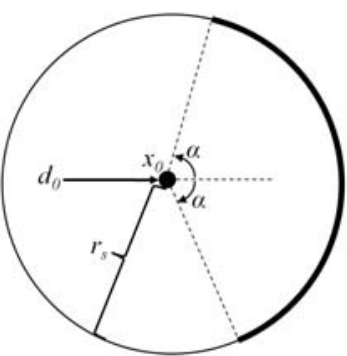

(c)

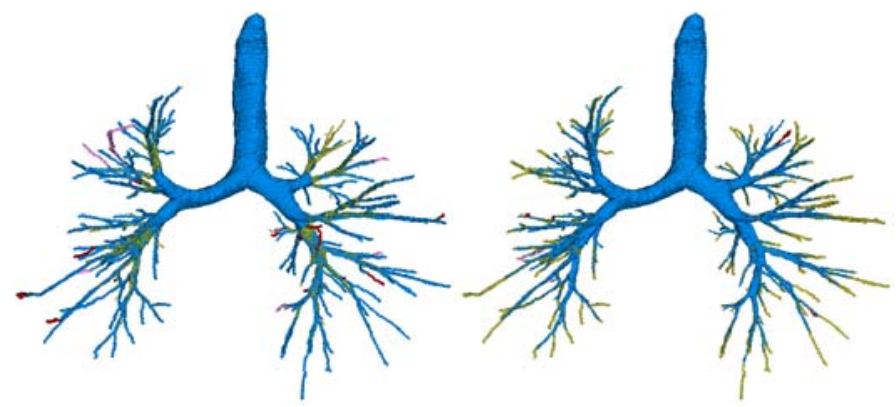

(d)

Fig. 1. (a) Example of a case where it is not possible to segment the correct part (the largest part of the branch) from the leakage, with the branch segment highlighted in yellow and an arrow indicating the leakage. (b) A scatter plot of the tree length obtained by both methods for the 10 test cases. (c) A schematic of the search sphere. (d) Surface rendering of the results from proposed method (left) and region growing based method (right), with correct regions in blue, missing regions in yellow, partly wrong regions in purple and false positives in red.

randomly selected images were used for the training of the voxel classification based appearance model. The method was tested on an additional 10 randomly selected CT scans from different subjects.

Results were compared to a region growing based method that uses a trained airway appearance model and exploits the fact that orientation of airways and vessels are similar [5]. Airways branches from the second up to and including the third generation obtained by this method were used to initialize the proposed method. Since extraction of these first few branches is relatively easy, simpler methods such as intensity based region growing could be used for initialization instead.

The centerlines of the initial segmentation were used as the initial set of candidate points needed for the proposed method. A simplified version of the segmentation algorithm presented in [2], without any leakage detection, were used to extract the centerlines. The direction and radius associated to each candidate point is derived from the branch they belong to. For newly added candidate points, the radius is simply propagated directly from their parents. 


\subsection{Parameter and Settings}

For extracting the optimal paths, the radius of the search sphere was set to $r_{s}=20 \mathrm{~mm}$ and the maximum allowed angle to $\alpha=100$ degrees. In selection of the optimal paths, at most $N=30$ percent of points on the path may have a confidence below $\beta=0.5$, the straightness measure $\gamma=1.05$, minimum required distance between selected optimal paths $\delta=3 \mathrm{~mm}$. The maximum number of selected paths from a single candidate point $N_{\max }$ was set to 2 .

For the appearance model, $K=21$ was used for the KNN classifier and 7 scales distributed exponentially from 0.5 to $3.5 \mathrm{~mm}$ were used both to generate the features (refer to [5] for details) and for the multi-scale Hessian eigen analysis.

To prevent paths from growing into border of the lungs, candidate points that were within $3 \mathrm{~mm}$ of the lung surface were not evaluated.

\subsection{Results}

All results were evaluated manually by an experienced observer using the dedicated airway segmentation evaluation tool developed for the EXACT'09 airway extraction challenge [14. The results were divided into individual branches using the simplified version of [2], without any leakage detection. Airway branches are subsequently visually inspected and classified into the categories 'correct', 'wrong', or 'partly wrong' based on several views obtained from different cut planes at different angles and positions. Branches are said to be correct, if there is no leakage visible at all. A branch is said to be wrong, if the majority (more than $50 \%$ ) of it is leakage, and partly wrong, if the majority is correct.

As the evaluation tool was designed for evaluating segmentation and not centerlines, results from the proposed method were dilated in order to make them compatible with the evaluation tool.

As the evaluation process only separates branches at bifurcations, we are unable to isolate leakage from a branch, if it extends from the endpoints and does not bifurcate. This usually occurred at the end segments, where a whole branch is classified as 'partly wrong' due to a small amount of leakage at the end. Figure 1(a) shows an example of this situation. All partly wrong regions were excluded from further evaluation.

Compared to the results from the region growing approach, we observe a significant $(p<0.01)$ increase of $36 \%$ in total tree length for the results of the proposed method, from an average of $192 \mathrm{~cm}$ tree length to $258 \mathrm{~cm}$. The tree length is defined as the sum of the lengths of all branches obtained, excluding the trachea and main bronchi. The average false positive rate, computed as a percentage of the total tree with the trachea and main bronchi excluded, was $3.79 \%$ for the proposed method, slightly larger than for the region growing based method (1.35\%). The total number of branches extracted was similar for both methods, around 174 branches on average. A plot showing the tree length for the 10 cases from the two methods are given in Fig. 1(b). Surface renderings of a randomly selected case are given in Fig. $1(\mathrm{~d})$

Finally, a third segmentation was constructed as the union of the airway trees extracted from the two methods. A comparison of the union against the result 
from the region growing based method shows a significant $(p<0.01)$ increase of $9 \%$ in branch count. Results from the proposed method cover $96 \%$ of the tree length in the union, while results from the region growing based method only have $71 \%$ coverage.

\section{Discussion and Conclusions}

The proposed method improves results significantly, especially in terms of tree length, where an average improvement of $36 \%$ was observed. The branches missed by the proposed method that were extracted correctly using the region growing based method were very short, with an average length of $5 \mathrm{~mm}$. Despite having similar branch count when comparing the proposed method and the region growing based method, significant increase (9\%) in branch count was observed when comparing the results from the region growing based method against the union. This indicates that the proposed method is capable of obtaining new branches, and not only merely extending branches obtainable by the region growing based method. It should be noted that the multi-scale version of the work from [5], used for comparison, is a method that gives results comparable to other recent state of the art methods such as [4].

In the current work, the estimate of the airway radius is propagated from old to new candidate points unaltered. Although this estimate is only used to determine the upper bound in the scale selection of Section 3 , more reliable estimates of local tubeness and tube orientation may be found if the radius is updated with each bifurcation to reflect the overall decrease in airway diameter towards the higher generations. Alternatively, an airway wall segmentation algorithm such as 15. could be used to obtain the radius, with the additional advantage that the result can be directly used for airway dimension analysis.

Another potential improvement would be to introduce a "multi-radius" scheme, which involves searching paths within multiple search spheres of different radii. This might increase the number of new branches found, as shorter branches may then be extracted using a smaller radius, while occlusions can still be overcome using the larger radii.

In conclusion, an airway tree extraction method that is based on a recursive search for locally optimal paths is presented. In contrast to common airway tree segmentation methods that only consider the immediate neighbors of seed points, our method considers both the appearance and structure of a whole path. This enables the method to extend past local occlusions caused by noise or pathologies such as mucus plugging. The proposed method handles bifurcations automatically, which is a topic rarely touched upon by optimal path tracking methods.

Acknowledgments. This work is partly funded by the Danish Council for Strategic Research (NABIIT), the Netherlands Organization for Scientific Research (NWO), and AstraZeneca, Lund, Sweden. 


\section{References}

1. Berger, P., Perot, V., Desbarats, P., de Lara, J.M.T., Marthan, R., Laurent, F.: Airway wall thickness in cigarette smokers: quantitative thin-section CT assessment. Radiology 235(3), 1055-1064 (2005)

2. Schlathölter, T., Lorenz, C., Carlsen, I.C., Renisch, S., Deschamps, T.: Simultaneous segmentation and tree reconstruction of the airways for virtual bronchoscopy. In: Sonka, M., Fitzpatrick, J.M. (eds.) SPIE Medical Imaging, vol. 4684, pp. 103113. SPIE, San Jose (2002)

3. Tschirren, J., Hoffman, E., McLennan, G., Sonka, M.: Intrathoracic airway trees: segmentation and airway morphology analysis from low-dose CT scans. IEEE T. Med. Imaging 24(12), 1529-1539 (2005)

4. van Ginneken, B., Baggerman, W., van Rikxoort, E.: Robust segmentation and anatomical labeling of the airway tree from thoracic CT scans. In: Metaxas, D., Axel, L., Fichtinger, G., Székely, G. (eds.) MICCAI 2008, Part I. LNCS, vol. 5241, pp. 219-226. Springer, Heidelberg (2008)

5. Lo, P., Sporring, J., Ashraf, H., Pedersen, J., de Bruijne, M.: Vessel-guided airway segmentation based on voxel classification. In: Brown, M., de Bruijne, M., van Ginneken, B., Kiraly, A., Kuhnigk, J., Lorenz, C., Mori, K., Reinhardt, J. (eds.) Proc. of First International Workshop on Pulmonary Image Analysis (2008)

6. Jbabdi, S., Bellec, P., Toro, R., Daunizeau, J., Pélégrini-Issac, M., Benali, H.: Accurate anisotropic fast marching for diffusion-based geodesic tractography. Journal of Biomedical Imaging 2008(1), 1-12 (2008)

7. Jackowski, M., Papademetris, X., Dobrucki, L.W., Sinusas, A.J., Staib, L.H.: Characterizing vascular connectivity from microCT images. In: Duncan, J.S., Gerig, G. (eds.) MICCAI 2005. LNCS, vol. 3750, pp. 701-708. Springer, Heidelberg (2005)

8. Gülsün, M.A., Tek, H.: Robust vessel tree modeling. In: Metaxas, D., Axel, L., Fichtinger, G., Székely, G. (eds.) MICCAI 2008, Part I. LNCS, vol. 5241, pp. 602 611. Springer, Heidelberg (2008)

9. Benmansour, F., Cohen, L.: Fast object segmentation by growing minimal paths from a single point on $2 \mathrm{D}$ or $3 \mathrm{D}$ images. Journal of Mathematical Imaging and Vision 33(2), 209-221 (2009)

10. Graham, M.W., Gibbs, J.D., Higgins, W.E.: Robust system for human airway-tree segmentation. In: SPIE Medical Imaging. SPIE, vol. 6914, 69141J (2008)

11. Kittler, J., Alkoot, F.M.: Moderating k-NN classifiers. Pattern Analysis \& Applications 5(3), 326-332 (2002)

12. Lindeberg, T.: Feature detection with automatic scale selection. Int. J. Comput. Vision 30(2), 79-116 (1998)

13. Pedersen, J., Ashraf, H., Dirksen, A., Bach, K., Hansen, H., Toennesen, P., Thorsen, H., Brodersen, J., Skov, B., Døssing, M., Mortensen, J., Richter, K., Clementsen, P., Seersholm, N.: The Danish randomized lung cancer CT screening trial - overall design and results of the prevalence round. Journal of Thoracic Oncology (April 2009)

14. Lo, P., van Ginneken, B., Reinhardt, J., de Bruijne., M.: Extraction of airways from CT 2009 (EXACT 2009). In: Proc. of Second International Workshop on Pulmonary Image Analysis (under preparation 2009)

15. Kiraly, A.P., Odry, B.L., Naidich, D.P., Novak, C.L.: Boundary-specific cost functions for quantitative airway analysis. In: Ayache, N., Ourselin, S., Maeder, A. (eds.) MICCAI 2007, Part I. LNCS, vol. 4791, pp. 784-791. Springer, Heidelberg (2007) 